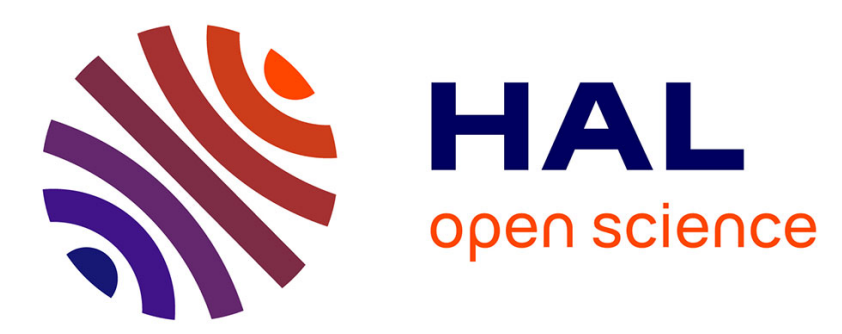

\title{
Multi-objective optimization of the induction machine with minimization of audible electromagnetic noise
} Jean Le Besnerais, Michel Hecquet, Vincent Lanfranchi, Pascal Brochet

\section{To cite this version:}

Jean Le Besnerais, Michel Hecquet, Vincent Lanfranchi, Pascal Brochet. Multi-objective optimization of the induction machine with minimization of audible electromagnetic noise. European Physical Journal: Applied Physics, 2007. hal-01713833

\section{HAL Id: hal-01713833 \\ https://hal.science/hal-01713833}

Submitted on 20 Feb 2018

HAL is a multi-disciplinary open access archive for the deposit and dissemination of scientific research documents, whether they are published or not. The documents may come from teaching and research institutions in France or abroad, or from public or private research centers.
L'archive ouverte pluridisciplinaire HAL, est destinée au dépôt et à la diffusion de documents scientifiques de niveau recherche, publiés ou non, émanant des établissements d'enseignement et de recherche français ou étrangers, des laboratoires publics ou privés. 


\title{
Multi-objective optimization of the induction machine with minimization of audible electromagnetic noise
}

\author{
Jean Le Besnerais ${ }^{1}$, Michel Hecquet ${ }^{1}$, Vincent Lanfranchi ${ }^{2}$, and Pascal Brochet ${ }^{1}$ \\ 1 L2EP, Ecole Centrale de Lille, 59651 Villeneuve d'Ascq, France (email: jean.le_besnerais@centraliens.net) \\ 2 LEC, Compiègne, France (email: vincent.lanfranchi@utc.fr)
}

Received: 19 January 2007/ Revised version: 01 March 2007

\begin{abstract}
Induction motors optimal design can involve many variables and objectives, and generally requires to make several trade-offs, especially when including the audible electromagnetic noise criterion beyond the usual performance criteria. Multiobjective optimization techniques based on Pareto optimality are useful to help us finding the most interesting solutions and decide which one(s) to adopt. However, it is not always easy to analyse the Pareto-optimal solutions obtained with such methods, especially when treating more than three objectives, and Pareto fronts may contain more data than we might think. This paper briefly describes an analytical model of the variable-speed squirrel-cage induction machine which computes both its performances and sound power level of electromagnetic origin. The model is then coupled to the Non-dominated Sorting Genetic Algorithm (NSGA-II) in order to perform global optimization with respect to several objectives (e.g. noise level, efficiency and material cost). Finally, an optimization problem is solved and analysed, and some useful visualization tools of the Pareto optimal solutions and their characteristics are presented.
\end{abstract}

PACS. 02.60.Pn Numerical optimization - 07.05.Tp Computer modeling and simulation - 07.50.-e Electrical and electronic instruments and components - 43.50.Ed Noise generation

\section{Nomenclature}

$D_{c} \quad$ stator outer diameter (without frame)

$g \quad$ air-gap width

$G \quad$ number of generations

$h_{c}$ height of stator yoke

$h_{f}$ height of stator frame

$L_{c} \quad$ stator stack length

$L_{f} \quad$ stator frame length

$l_{\text {se }} \quad$ stator slot opening

$l_{\text {re }} \quad$ rotor slot opening

$M \quad$ number of design variables

$N \quad$ number of objectives

$P \quad$ population size

$R_{f} \quad$ stator frame mean radius

$\alpha_{s} \quad$ angular position in stator steady frame

$\eta \quad$ motor efficiency at nominal speed

\section{Introduction}

Induction machine optimal design is an issue which has received much attention since the beginning of computer science [1-8]. It usually aims at reaching some given performances with high efficiency and low cost. However, as acoustic norms become stricter in electric transport systems, the noise of electromagnetic origin which comes from the air-gap Maxwell forces acting on the stator inner surface is another important factor to consider during optimization. We now aim at designing induction machines with high efficiency, high torque, high specific power, low material cost and low magnetic noise.

Dealing with the noise or vibration criterion in a multiobjective optimization of an electrical machine is rather recent, and the first work may come from C. Hadj Amor [9] who studied the trade-off between noise level and material cost. Many other trade-offs have to be made during induction machine design. For instance, a small air-gap is known to improve its efficiency, but it usually increases it sound power level. In a same way, decreasing the stator diameter to height of yoke ratio $D_{c} / h_{c}$ generally lowers the sound power level, but leads to a smaller volume available for the rotor, and therefore to a higher temperature and lower efficiency [9]. The machine ability to radiate noise also decreases with the ratio $R_{f} / L_{f}$, where $L_{f}$ is the stator frame length and $R_{f}=D_{c} / 2+h_{f} / 2$ its mean radius [10]. These are general considerations, and the motor geometry influence on noise is more complex: the sound power level of electromagnetic origin depends on the match between the motor natural modes and frequencies and the magnetic exciting forces modes and fequencies [11]. Finally, the motor geometry is of course directly linked to the motor material cost. 
When dealing with several conflicting objectives, using Pareto optimality based algorithms like the Non-dominated Sorting Genetic Algorithm (NSGA-II) [12] allows not to obtain a single optimal solution, but a large set of Pareto-optimal solutions. At the final stage of the optimization process, it makes possible for the designer to add some new criteria and then make a final decision.

This article first presents the electromagnetic, mechanical and acoustic models used during the optimization procedure. Then, the NSGA-II optimization method is described and some optimization results are presented and analysed. Finally, some useful visualization tools of Pareto optimal solutions are presented.

\section{Electromagnetic, mechanical and acoustic models}

In order to compute the audible electromagnetic sound power level radiated by an induction machine, some electromagnetic, mechanical and acoustic models are coupled. As the goal is to perform evolutionary optimizations, these models must have a low computational complexity. Therefore, some analytical models are used and validated with tests and finite elements simulations [13,14]. Some rather simple models can be used within the optimization framework as long as they give the right relative trends.

\subsection{Electromagnetic model}

The stator and rotor currents computation are based on a fundamental single phase equivalent circuit. This circuit computes the motor performances such as efficiency and output torque. The stator and rotor magnetomotive forces $f_{m m}^{s}$ and $f_{m m}^{r}$ are computed by the aid of the winding functions [15]. However, that are not expressed as Fourier series in order to lower computation times. Then, the permeance per unit area $\Lambda$ is computed and the radial air-gap flux density $B_{g}$ is expressed as:

$$
B_{g}\left(t, \alpha_{s}\right)=\Lambda\left(t, \alpha_{s}\right)\left(f_{m m}^{s}\left(t, \alpha_{s}\right)+f_{m m}^{r}\left(t, \alpha_{s}\right)\right)
$$

Note that the saturation effects as well as iron losses are not considered in the simulations presented in this article.

\subsection{Mechanical model}

Neglecting the tangential component of the Maxwell tensor and the magnetostrictive effect, the radial exciting pressure $F_{r}$ which is supposed to be responsible for magnetic noise can be approximated by

$$
F_{r}=B_{g}^{2} /\left(2 \mu_{0}\right)
$$

where $\mu_{0}$ is the air-gap magnetic permeability. The static radial displacements $Y_{s}^{m}$ are first computed from the complex amplitudes $F_{m w}$ of the 2D discrete Fourier transform
(FFT2) of $F_{r}$. Then, the dynamic displacements $Y_{d}^{m}$ are computed by the aid of a the magnification factor:

$$
Y_{d}^{m}=Y_{s}^{m}\left[\left(1-f^{2} / f_{m}^{2}\right)^{2}+4 \xi_{m}^{2} f^{2} / f_{m}^{2}\right]^{-1 / 2}
$$

where $\xi_{m}$ is the modal damping coefficient, and $f_{m}$ is the $m$-th mode natural frequency. These frequencies are computed assuming that the stator is a $2 \mathrm{D}$ ring [10]. $\xi_{m}$ can be computed using the experimental law established by $[16]$

$$
2 \pi \xi_{m}=2.76 \times 10^{-5} f_{m}+0.062 \Rightarrow \xi_{m} \in[1 \%, 3 \%]
$$

The vibration velocity of mode number $m$ is finally given by $v_{m}=Y_{d}^{m} 2 \pi f$.

\subsection{Acoustic model}

The sound power radiated by the vibrations of mode $m$ and frequency $f$ is

$$
W_{m}(f)=\rho_{0} c_{0} S_{c} \sigma_{m}<\overline{v_{m}^{2}}>
$$

where $S_{c}$ is the stator outer surface, $\rho_{0}$ the air density, $c_{0}$ the speed of sound, and $\sigma_{m}$ the modal radiation efficiency. $\sigma_{m}$ is approximated using either its pulsating sphere expression or its infinite cylinder expression according to the stator dimensions [17].

The total sound power level is finally

$$
L_{w}=10 \log _{10}\left(\sum_{f, m} W_{m}(f) / W_{0}\right), \quad W_{0}=10^{-12} W
$$

More details about these electromagnetic, mechanical and acoustic models and their validation can be found in $[14,18]$.

\section{Non-dominated Sorting Genetic Algorithm}

We consider a vector of objective functions to minimize $\mathbf{F}=\left(f_{1}, \ldots, f_{N}\right)$ which depend on a design variables vector $\mathbf{X}=\left(X_{1}, \ldots, X_{M}\right)$. The unconstrained optimization problem can be written under the form

$$
\min \mathbf{F}(\mathbf{X})
$$

Aggregating optimization techniques replace the problem (7) by

$$
\min \sum_{i=1}^{N} \lambda_{i} f_{i}(\mathbf{X})
$$

where $\lambda_{i}$ represents the weight given by the designer to the $i$-th objective. However, giving to the weights the right value is a tough task, and the aggregating method lead to a single solution which make impossible to add other criteria (e.g. temperature when simulations do not 
include a thermal model) or empirical knowledge at the end of the optimal design search. On the contrary, multiobjective optimization algorithms do not try to solve $a$ priori the conflicting objectives: they provide a whole set of solutions among which the designer can a posteriori choose.

NSGA-II is one of the most efficient multi-objective evolutionary algorithms using an elitist approach $[12,19]$. The current population offspring is obtained using a binary tournament selection and the standard bimodal crossover and polynomial mutation operators used in genetic algorithms. Then, in order to form the next generation from the combined current population and offspring, NSGA-II algorithm uses a particular fitness assignment scheme which consists in sorting the individuals in different fronts using the non-domination order relation (Fig. 1).

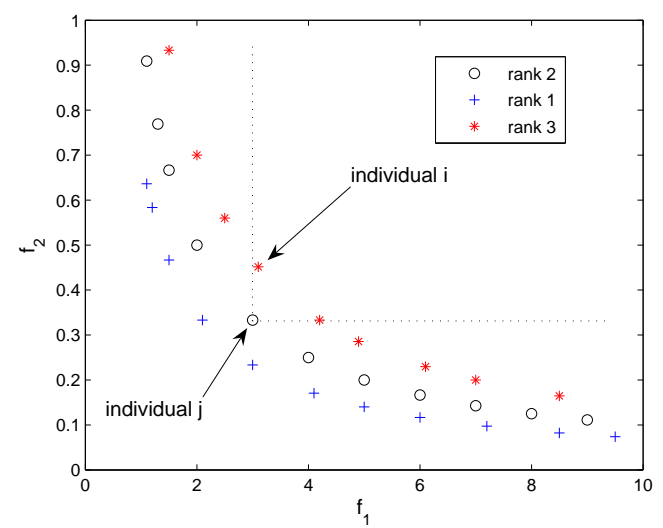

Fig. 1. Illustration of NSGA ranking method in case of a 2-D Pareto front. Individual $j$ is non-dominated by $i$ if there exist $k \in[1, N]$ such as $f_{k}^{j}<f_{k}^{i}, f_{k}^{i}$ denoting the $k$-th objective function of individual $i$.

The new population is then chosen in terms of nondominance and diversity.

\section{Definition of the optimization problem}

\subsection{Design variables}

We here consider the $M=8$ continuous geometric design variables $h_{c}, D_{c}, L_{f}, h_{f}, l_{s e}, l_{r e}, L_{c}$ and $g$ (see FIG. 2). Note that the active stator stack length $L_{c}$ and the stator frame length $L_{f}$ are supposed to be different: $L_{f}$ influences the motor ability to radiate noise whereas $L_{c}$ influences its electromagnetic performances.

\subsection{Objective functions}

The definition of the objective functions is very important because it has much influence on the optimization results. Five possible definitions of the variable-speed noise objective function are here presented. A first idea consists in

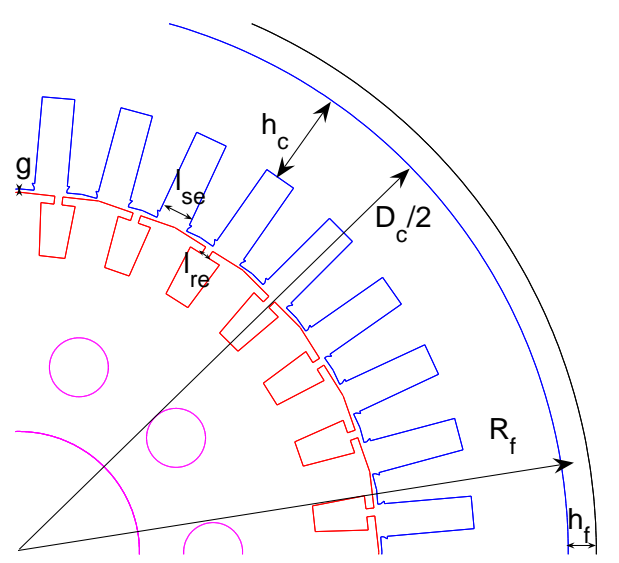

Fig. 2. Induction machine main geometric variables.

computing the noise level at the motor nominal frequency $f_{s}^{N}$ :

$$
L_{w}^{1}=L_{w}\left(f_{s}^{N}\right)
$$

Minimizing $L_{w}^{1}$ will therefore significantly reduce the noise level at the most important supply frequency. In the same way, we could define

$$
L_{w}^{2}=\frac{\int_{f_{s}} L_{w}\left(f_{s}\right) w\left(f_{s}\right) d f_{s}}{\int_{f_{s}} w\left(f_{s}\right) d f_{s}}
$$

where $w\left(f_{s}\right)$ represents the machine lifecycle proportion at frequency $f_{s}$. In that case, minimizing $L_{w}^{2}$ will lead to a quieter machine during its whole lifecycle, and noise will be mostly reduced at nominal frequency. A third definition consists in computing the average noise level on the whole machine speed range:

$$
L_{w}^{3}=\frac{\int_{f_{s}} L_{w r}\left(f_{s}\right) d f_{s}}{\int_{f_{s}} d f_{s}}
$$

If some noise specifications $L_{s p}\left(f_{s}\right)$ must be fulfilled, one can use:

$$
L_{w}^{4}=\max _{f_{s}}\left\{L_{w}\left(f_{s}\right)-L_{s p}\left(f_{s}\right)\right\}
$$

Finally, one can also use the maximum level of noise encountered in the whole speed range:

$$
L_{w}^{5}=\max _{f_{s}} L_{w}\left(f_{s}\right)
$$

All these different definitions can be used for any variable-speed objective. As explained in [14], using $L_{w}^{1}$ can lead to irrelevant solutions: the noise level is artificially reduced by putting the machine natural frequencies away from the exciting force frequencies at the given supply frequency $f_{s}^{N}$, and harmful resonances can still occur close to $f_{s}^{N}$. Moreover, using $L_{w}^{4}$ is not interesting as it is based on 


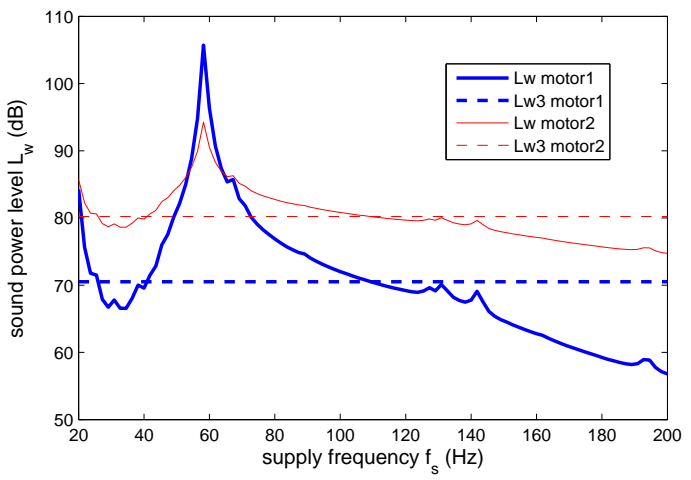

Fig. 3. Variable-speed noise level of two different designs in function of supply frequency. This figure illustrates the fact that motor1 design has a lower $L_{w}^{3}$ but a higher $L_{w}^{5}$ than motor2 design.

the comparison between the real noise level $L_{s p}$ which necessarily includes noise of mechanical and aeraulic sources (e.g. bearings and fans), and a simulated magnetic noise level $L_{w}$. In this paper, the noise objective functions that we consider are both $L_{w}^{5}$ which ensures minimization of the resonance levels and $L_{w}^{3}$ which ensures minimization of the average noise (see Fig. 3). These two objective functions could be aggregated defining the following new objective function:

$$
L_{w}^{6}=10 \log \left(10^{0.1 L_{w}^{3}}+10^{0.1 L_{w}^{5}}\right)
$$

We here consider $N=5$ objective functions to minimize: the average sound power level $L_{w}^{3}$ in $\mathrm{dB}$, the maximum sound power level $L_{w}^{5}$ in $\mathrm{dB}$, material cost $P_{r}$ in euros, mass per unit of output power $M_{k w}$ in $\mathrm{kg} / \mathrm{W}$ (inverse of specific power) and the motor inverse efficiency $1-\eta$ at nominal speed $(85 \mathrm{~Hz})$. Material cost is computed evaluating the amount of iron, copper and aluminium in the machine. Efficiency is computed as:

$$
\eta=\frac{P_{\text {out }}}{P_{\text {in }}}=\frac{P_{\text {out }}}{P_{\text {gap }}+P_{j s}} ;
$$

where $P_{\text {out }}$ is the mechanical output power (neglecting the mechanical friction losses and iron losses), $P_{\text {gap }}$ is the air-gap electromagnetic power, and $P_{j s}$ is the stator Joule power losses. All these quantities are computed using the single-phase equivalent circuit. Finally, $M_{k w}$ is simply computed as $m / P_{\text {out }}$ where $m$ is the machine total mass.

\subsection{Constraints}

A repair algorithm is used in order to fix the individuals which do not respect geometric constraints (for instance, too large stator slot openings $l_{\text {se }}$ lead to negative stator teeth width). However, we do not consider any design constraints in this paper.

\section{Optimization results}

Table 1. Optimization problem OP1

\begin{tabular}{|c|c|}
\hline Design variables & $h_{c}, D_{c}, L_{f}, h_{f}, l_{s e}, l_{r e}, L_{c}, g$ \\
Objective functions & $P_{r}, L_{w}^{3}, L_{w}^{5}, M_{k w}, 1-\eta$ \\
\hline
\end{tabular}

We consider the optimization problem of Tab. 1 . The initial population is made of $P=100$ individuals randomly generated in some specified design variables bounds. The distribution indices for mutation and cross-over operators are fixed to 30 , and the number of generations is $G=50$. The noise objective function is computed in offload sinusoidal case on the speed range $f_{s} \in[10 \mathrm{~Hz}, 100$ $\mathrm{Hz}$ ] (traction mode at constant flux). The speed range discretization step is fixed to $\Delta f_{d}=3 \mathrm{~Hz}$, i.e. noise level is computed at 30 different supply frequencies. $\Delta f_{d}$ is fixed in order to correctly take into account resonance phenomena. If a resonance is defined by a $\pm 3 \mathrm{~dB}$ variation of the noise level around the natural frequency $f_{m}$, we must have:

$$
\Delta f_{d}<\min _{m} 2 \xi_{m} f_{m}
$$

As $\xi_{m} \geq 1 \%$ and $f_{m} \geq 250 \mathrm{~Hz}$, we obtain the condition $\Delta f_{d}<5 \mathrm{~Hz}$ which is fullfilled with $\Delta f_{d}=3 \mathrm{~Hz}$. One evaluation of the variable-speed objective function takes about $t_{1}=10 \mathrm{~s}$, and the NSGA-II algorithm runs in approximately $10 \mathrm{~h}$ with a $2.13 \mathrm{GHz}$ processor.

The evolution of best fitnesses during NSGA-II iterations is shown in Fig. 4.

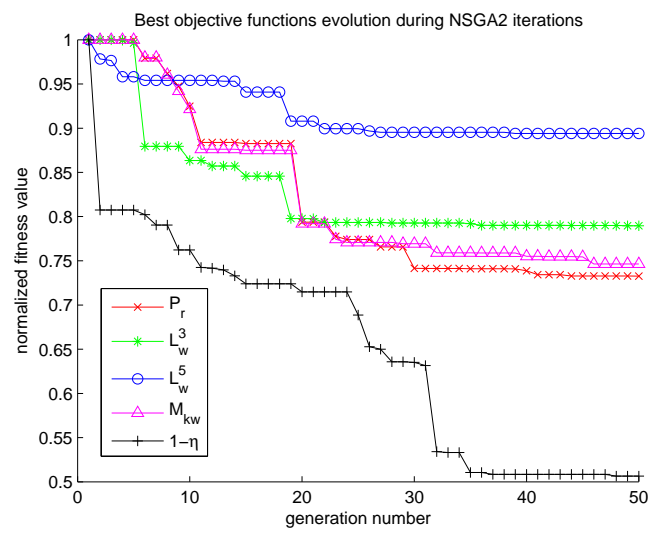

Fig. 4. Evolution of NSGA-II current population best fitnesses during the 50 generations (OP1 problem).

When dealing with more than three objectives, it becomes impossible to visualize the whole Pareto front in a single figure. However, it is always possible to draw all its 3-D or 2-D projections.

The $3 \mathrm{D}$ projection in space $\left(L_{w}^{3}, P_{r}, 1-\eta\right)$ is shown in Fig. 5. It has been materialized with a Delaunay triangularization, and the individuals have been projected on the corresponding three hyperplanes. The objective function 


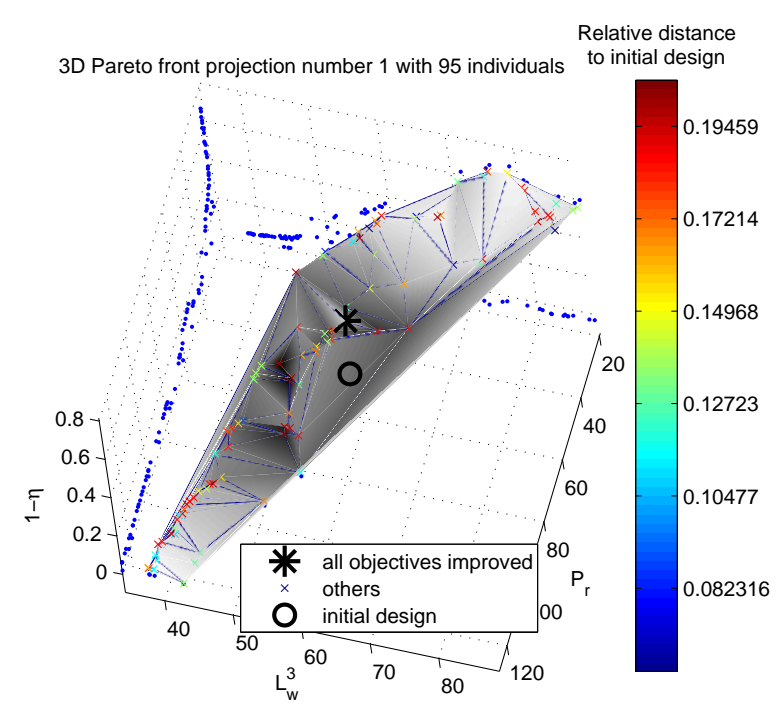

Fig. 5. Final Pareto front projection in $\left(L_{w}^{3}, P_{r}, 1-\eta\right)$ space (OP1 problem). Only 95 individuals out of 100 are plotted because individuals which do not respect geometric constraints are not represented. The projection of the Pareto front on the 3 planes have been also plotted.

of an existing industrial design has been plotted to be compared with simulation results. The Pareto Front individuals have been compared to this initial design, and only one individual among the 100 improves simultaneously all the objective functions of the existing design. This is because we have considered many objectives: indeed, 14 individuals exactly improve 4 objectives, 55 individuals improve 3 of them and 30 improve 2 of them. Moreover, the initial population was widespread in the design variables space ( $\pm 30 \%$ of the existing design dimensions).

In fact, if the optimization aims at finding a design $\mathbf{X}$ improving an existing one $\mathbf{X}_{\mathbf{0}}$, one can run NSGA-II adding in the objective function computation $\mathbf{F}_{\mathbf{c}}$ a penaltybased constraint:

$$
\mathbf{F}_{\mathbf{c}}(\mathbf{X})=\mathbf{F}(\mathbf{X})+\mathbf{C}\left(\mathbf{X}, \mathbf{X}_{\mathbf{0}}\right)
$$

where

$C_{i}\left(\mathbf{X}, \mathbf{X}_{\mathbf{0}}\right)=\left\{\begin{array}{l}K_{i}\left(f_{i}(\mathbf{X})-f_{i}\left(\mathbf{X}_{\mathbf{0}}\right)\right) \\ 0 \quad \text { otherwise }\end{array} \quad\right.$ if $\quad f_{i}(\mathbf{X})>f_{i}\left(\mathbf{X}_{\mathbf{0}}\right)$

There are several ways to choose the $K_{i}$ factors quantifying the severity of $f_{i}(\mathbf{X}) \leq f_{i}\left(\mathbf{X}_{\mathbf{0}}\right)$ constraint. It can be fixed to 1 independently of the generation number $t$, or it can be increased during generations (for instance $K_{i}=t$ ) in order to allow highly infeasible solutions at the beginning of the search, and end it with feasible designs. More details about these methods can be found in [20]. Using this penalization technique, far more individuals improving all the existing design objectives can be obtained.

In Fig. 5, the Pareto front individuals have been coloured in function of their Euclidian distance to the existing design in the design variables space. It helps visualizing data coming from both the objective functions space and the design variables space (through the colours legend).
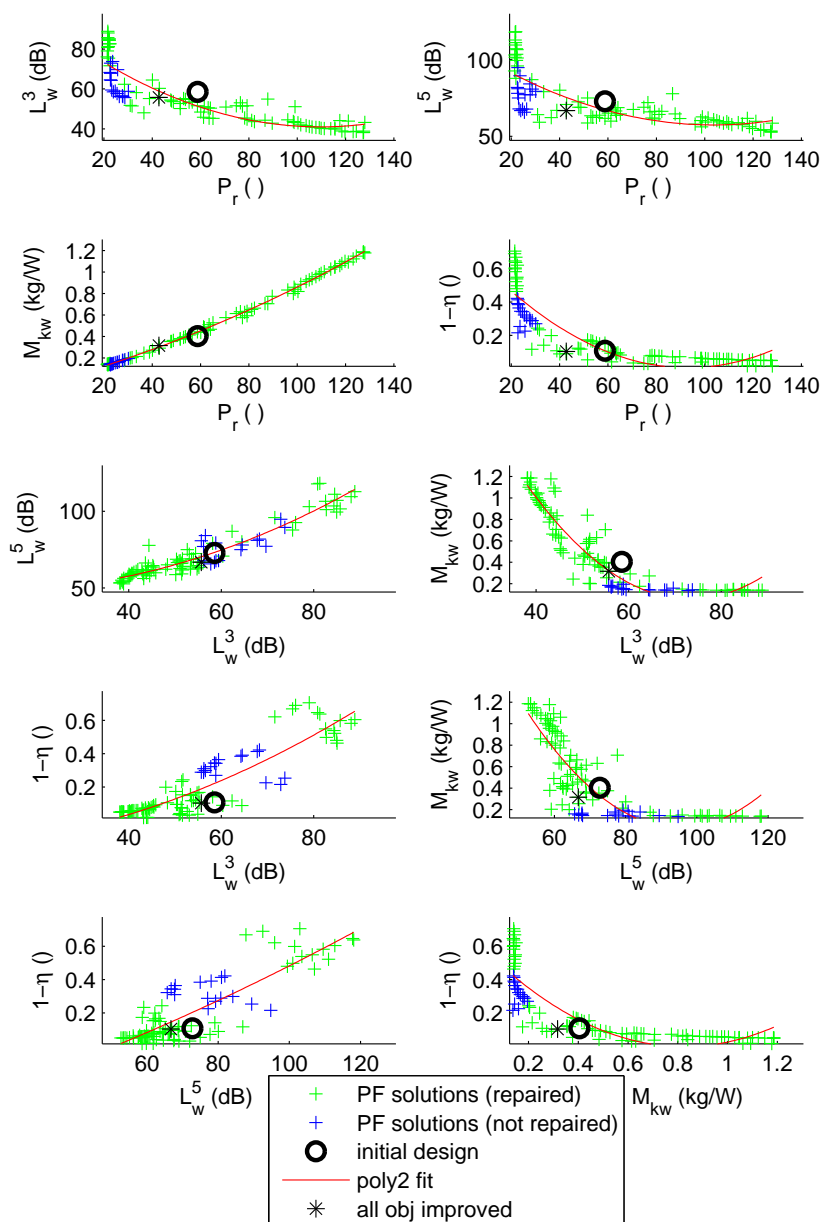

Fig. 6. Final population (Pareto front) 2-D projections (OP1 problem).

In addition, it allows distinguishing innovative designs, which are very different from the existing one and may require new manufacturing process, from designs representing slight modifications of the existing one.

All the Pareto front 2-D projections are plotted in Fig. 6. As we have 5 objectives, we obtain $\left(\begin{array}{l}5 \\ 2\end{array}\right)=10$ different projections. The trade-offs curves in planes $\left(L_{w}^{3 / 5}, P_{r}\right)$, $\left(1-\eta, P_{r}\right),\left(1-\eta, M_{k w}\right)$ and $\left(M_{k w}, L_{w}^{3 / 5}\right)$ have the same convex shape. They show for instance that we cannot minimize at the same time noise level and material price. In a same way, maximizing efficiency leads to a higher cost and a lower specific power. The projections $\left(M_{k w}, P_{r}\right)$ and $\left(L_{w}^{5}, L_{w}^{3}\right)$ have the same linear shape. They show that minimizing the average noise globally leads to a lower maximum noise, and that minimizing material cost generally leads to a higher specific power. The two projections $\left(1-\eta, L_{w}^{3 / 5}\right)$ are more scattered, which means that we can nearly minimize $1-\eta$ and $L_{w}^{3 / 5}$ independently. In all these figures, the individuals that were "repaired" during the evaluation of the objective function, i.e. which did not respect basic geometric constraints, are displayed in a different color than the individuals which did not need to be repaired. There are obviously many individu- 
als representing non-feasible designs: without any repair algorithm, all these individuals would be assigned a high fitness value in order to avoid inconsistent geometries in the final Pareto front, which would degrade population diversity and NSGA-II convergence.

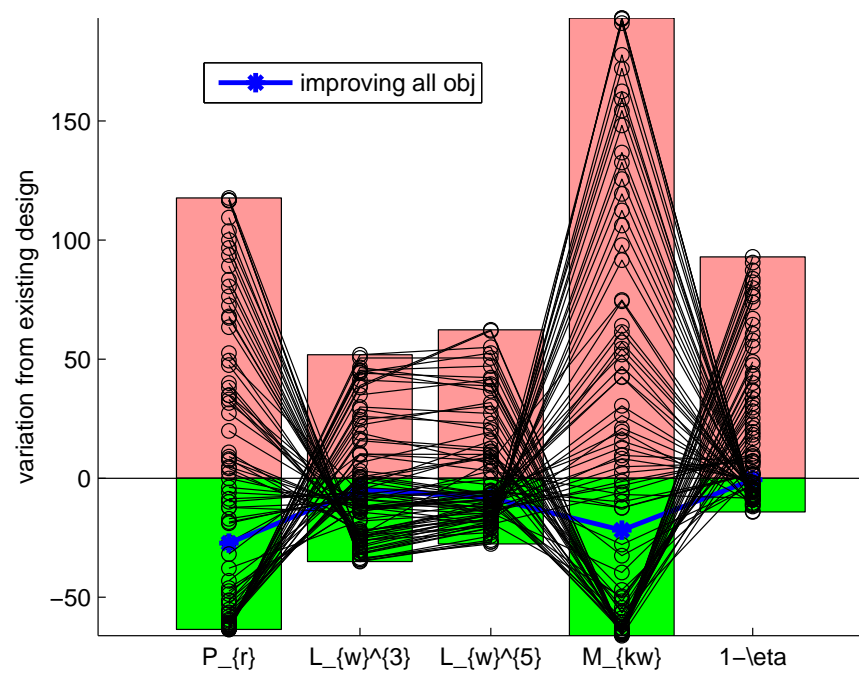

Fig. 7. Variation of objective functions with respect to initial design (OP1 problem). Some Pareto-optimal solutions are not represented for readability. The individual improving all the objectives reaches $[-25 \%,-5 \%,-9 \%,-21 \%,-3 \%]$.

Fig. 7 represents the objective functions variations with respect to the existing design. The only individual improving all the existing design objectives reaches a $25 \%$ material cost decrease, a $5 \%$ average noise decrease, a $9 \%$ maximum noise decrease, a $21 \%$ increase in specific power, and a $3 \%$ efficiency increase. In this figure, we can also easily see the trade-offs to be made: for example, the individuals with the lowest noise levels have the highest material cost and lowest specific power.

Fig. 8 represents the objective functions of the 50 Pareto fronts generated during NSGA-II iterations in function of the design variables $D_{c}$ and $h_{c}$. It helps seeing if an objective is directly linked to a particular design variable. In this example, all the individuals are almost randomly distributed in the graphs. The only design variable whose influence on objective functions is clear is the stator diameter $D_{c}$ : when it increases, the maximum and average noise levels tend to slighly decrease, whereas material cost and mass per unit of power increase. The effect of $D_{c}$ on noise was not predictible: as explained in the introduction, increasing $D_{c}$ at constant stack length decreases it ability to radiate noise through the modification of its radiation factor. However, it also changes the stator natural frequencies, and increases vibration levels when keeping constant the height of yoke $h_{c}$.

The visibly high correlation between $D_{c}$ and the objective functions can be rigourously quantified using the

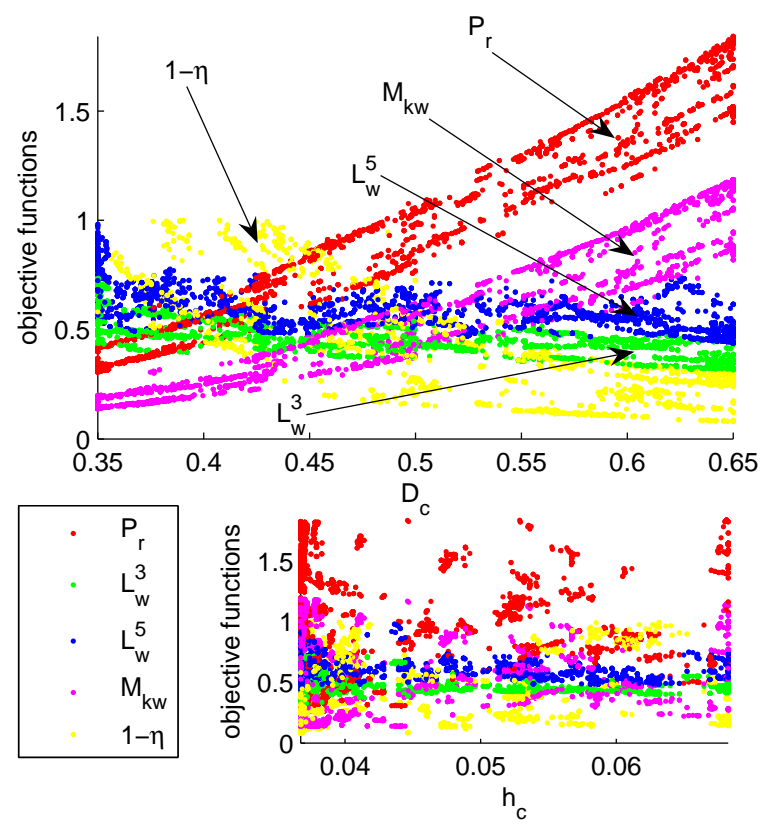

Fig. 8. Relation between design variables and objectives (OP1 problem).

correlation factor $\rho_{X Y}$ between variables $X$ and $Y$ :

$$
\rho_{X Y}=\frac{\operatorname{Cov}(X, Y)}{\sigma_{X} \sigma_{Y}} \in[-1,1]
$$

where $\operatorname{Cov}(X, Y)=E(X Y)-E(X) E(Y)$ is the covariance between $X$ and $Y$, and $\sigma_{X}$ is the standard deviation of $X$. Indeed, if $x_{i}$ and $f_{j}$ are higly correlated, for instance when $f_{j}=\alpha x_{i}$, we have $\rho_{x_{i} f_{j}}=1$. However, if they are independent, we have $\rho_{x_{i} f_{j}}=0$.

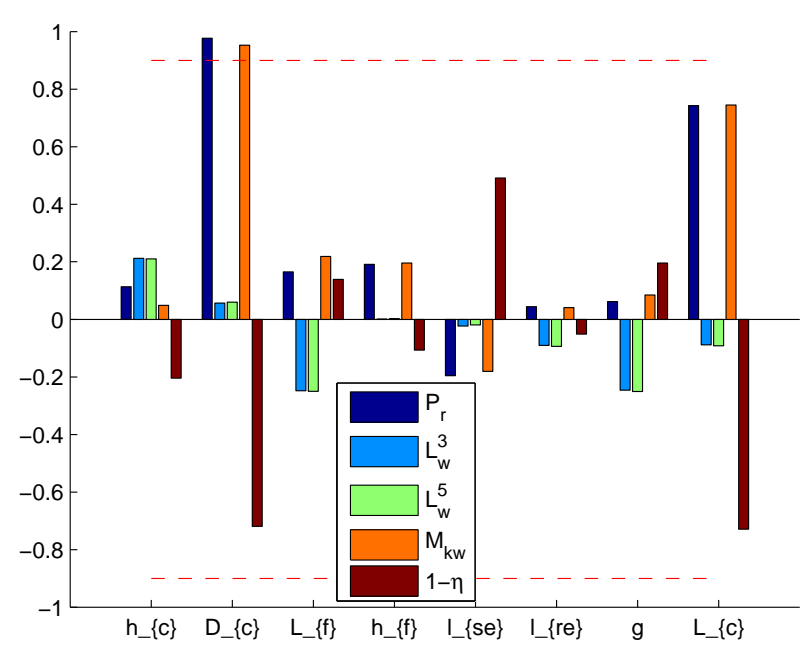

Fig. 9. Correlation factors between variables and objective functions (OP1 problem). $D_{c}$ is highly correlated to $P_{r}, M_{k w}$ and $1-\eta$.

Correlation factors are plotted in Fig. 9. The expectations are computed during all NSGA iterations in order to use all available data and obtain more accurate results. 
As previously seen in Fig. $8, D_{c}$ is the design variable the most correlated to objectives $M_{k w}, 1-\eta$ and $P_{r} . L_{c}$ is also significantly linked to the same objectives. The other design variables have low correlation factors, which shows that no simple design rule can be inferred.

\section{Conclusion}

This paper has shown how it is possible to design a cheap and efficient motor with a reasonable noise level of electromagnetic origin. A multi-objective genetic algorithm has been used in order to find some Pareto-optimal solutions achieving in particular low noise levels on a wide speed range. These new motor designs were reached by acting on both their natural frequencies and exciting electromagnetic forces. Simulations show that there is clearly a trade-off to make between material cost, specific power, efficiency and electromagnetic sound power level.

According to optimization results, it seems quite easy to improve a motor efficiency without degrading its quietness. Nethertheless, it is unrealistic because our model does not include the thermal criterion yet. Changing a motor's dimensions in order to avoid resonances and decrease its radiation factor can possibly alter the way it is cooled and raise temperature. Future work will therefore couple the existing model with a nodal-network thermal model in order to perform multi-objective optimizations including the thermal criterion. Constrained and mixed-variable multi-objective optimizations will be also investigated.

\section{References}

1. H. Huang, E. Fuchs, and Z. Zak, "Optimization of singlephase induction motor design," IEEE Transactions on Energy Conversion, vol. 3, no. 2, June 1988.

2. R. Fei, E. Fuchs, and H. Huang, "Comparison of two optimization techniques as applied to three-phase induction motor design," IEEE Transactions on Energy Conversion, vol. 4, no. 4, Dec. 1989.

3. X. Alabern and E. Valero, "Optimized design of an induction motor using fuzzy logic as a calculus and decision tool," IEEE Trans. on Electric Machines and Drives, June 2003.

4. G. Liuzzi, S. Lucidi, F. Parasiliti, and M. Villani, "Multiobjective optimization techniques for the design of induction motors," IEEE Trans. on Magnetics, vol. 39, no. 3, May 2003.

5. M. Nurdin, M. Poloujadoff, and A. Faure, "Synthesis of squirrel cage motors : a key to optimization," IEEE Transactions on E. C., vol. 6, no. 2, June 1991.

6. M. Poloujadoff, E. Christaki, and C. Bergniann, "Univariant search: An opportunity to identify and solve conflict problems in optimization," IEEE Transactions on Energy Conversion, vol. 9, no. 4, Dec. 1994.

7. M. Kim, D. Lee, and H. Jung, "Multiobjective design of three-phase induction motor using improved evolution strategy," IEEE Trans. on Magnetics, vol. 34, no. 5, Sept. 1998.
8. C. Singh and D. Sarkar, "Practical considerations in the optimisation of induction motor design," IEE Proceedings, vol. 139, no. 4, July 1992.

9. A. H. Amor, P. Timar, and M. Poloujadoff, "Induction squirrel cage machine design with minimization of electromagnetic noise," IEEE Transactions on Energy Conversion, vol. 10, no. 4, Dec. 1995.

10. J. Gieras, Noise of polyphase electric motors. CRC Press, Dec. 2005.

11. P. Timar, Noise and vibration of electrical machines. Elsever, 1989.

12. K. Deb, S. Agrawal, A. Pratap, and T. Meyarivan, "A fast elitist non-dominated sorting genetic algorithm for multiobjective optimization : NSGA-II," IEE Trans. on Evolutionary Computation, vol. 6, no. 2, Apr. 2002.

13. A. Ait-Hammouda, M. Hecquet, M. Goueygou, and P. Brochet, "Prediction of the electromagnetic noise of an asynchronous machine using experimental designs," Mathematics and Computers in Simulation, vol. 71, no. 4, June 2006.

14. J. L. Besnerais, A. Fasquelle, M. Hecquet, V. Lanfranchi, P. Brochet, A. Randria, D. Zorzynski, and S. Bujacz, "A fast noise-predictive multiphysical model of the PWMcontrolled induction machine," in Proceedings of the International Conference on Electrical Machines, Sept. 2006.

15. A. Ghoggal, M. Sahraoui, A. Aboubou, S. Zouzou, and H. Razik, "An improved model of the induction machine dedicated to faults detection - extension of the modified winding function approach," IEEE International Conference on Industrial Technology, Dec. 2005.

16. S. J. Yang, Low noise electrical motors. Clarendon Press, 1981.

17. P. Timar and J. Lai, "Acoustic noise of electromagnetic origin in an ideal frequency-converter-driven induction motor," IEE Proceedings on Electrical Power Applications, vol. 141 , no. 6, Nov. 1994.

18. J. L. Besnerais, V. Lanfranchi, M. Hecquet, and P. Brochet, "Acoustic noise of electromagnetic origin in a fractional-slot induction machine," in Proceedings of SSD'07, Feb. 2007, to be published.

19. M. Mohan, K. Deb, and S. Mishra, "A fast multi-objective evolutionary algorithm for finding well-spread Paretooptimal solutions," Kanpur Genetic Algorithms Laboratory (KanGAL), Tech. Rep., 2003.

20. A. E. Smith and D. W. Coit, Handbook of Evolutionary Computation. Oxford Press, 1995. 